\title{
African swine fever virus p37 structural protein is localized in nuclear foci containing the viral DNA at early post-infection times
}

\author{
Ana Eulálio ${ }^{\mathrm{a}, \mathrm{b}}$, Isabel Nunes-Correia ${ }^{\mathrm{a}, \mathrm{b}}$, José Salas ${ }^{\mathrm{c}}$, Maria L. Salas ${ }^{\mathrm{c}}$, \\ Sergio Simões ${ }^{\mathrm{a}, \mathrm{d}}$, Maria C. Pedroso de Lima ${ }^{\mathrm{a}, \mathrm{b}, *}$ \\ ${ }^{a}$ Center for Neuroscience and Cell Biology of Coimbra, University of Coimbra, 3004-517 Coimbra, Portugal \\ ${ }^{\mathrm{b}}$ Department of Biochemistry, Faculty of Sciences and Technology, University of Coimbra, 3001-401 Coimbra, Portugal \\ c Centro de Biología Molecular "Severo Ochoa” (Consejo Superior de Investigaciones Científicas-Universidad Autónoma de Madrid), \\ Universidad Autónoma, Cantoblanco, 28049 Madrid, Spain \\ ${ }^{\mathrm{d}}$ Department of Pharmaceutical Technology, Faculty of Pharmacy, University of Coimbra, 3000-295 Coimbra, Portugal \\ Received 17 January 2007; received in revised form 30 April 2007; accepted 4 May 2007 \\ Available online 18 June 2007
}

\begin{abstract}
The replication of African swine fever virus DNA is initiated inside the nucleus of host cells, being followed by a longer cytoplasmic replication stage. In face of previous results demonstrating the nucleo-cytoplasmic shuttling activity of ASFV p37 structural protein when considered isolated from the virus infection, we performed a systematic analysis of the subcellular localization of p37 protein in ASFV-infected cells, aiming at identifying the role of the nuclear transport mediated by this protein in the viral replication cycle. We report that the p37 protein of the incoming virions is localized throughout the cell at early times post-infection, concentrated in distinct nuclear regions, while at later times the newly synthesized protein is detected exclusively in the cytoplasm of infected cells. Experiments using leptomycin B and siRNAs targeting the CRM1 receptor demonstrate that the subcellular localization of p37 protein is not affected by inhibition of the CRM1-mediated nuclear export pathway. Finally, results from in situ hybridization experiments show a co-localization of the ASFV DNA and p37 protein in specific nuclear regions at early times post-infection, and in viral factories at later times. Overall, these results support the involvement of p37 protein in the nuclear transport of the viral DNA during ASFV replication cycle.
\end{abstract}

(C) 2007 Elsevier B.V. All rights reserved.

Keywords: African swine fever virus; p37 protein; CRM1-mediated nuclear export; CRM1-independent nuclear export; Nuclear replication stage

\section{Introduction}

African swine sever virus (ASFV), the only member of the new virus family Asfarviridae (Dixon et al., 2005), is the causative agent of a highly lethal hemorrhagic disease that affects domestic pigs (Vinuela, 1987).

The genome of the ASFV BA17V strain, a large linear double-stranded DNA molecule, has been completely sequenced and its analysis has identified 151 open-reading frames, which encode approximately 50 structural proteins and several enzymes involved in viral DNA replication, gene transcription and protein modification (Yanez et al., 1995). The

\footnotetext{
* Corresponding author at: Department of Biochemistry, Faculty of Sciences and Technology, University of Coimbra, Apartado 3126, 3001-401 Coimbra, Portugal. Tel.: +351 239820190; fax: +351 239853607.

E-mail address: mdelima@ci.uc.pt (M.C. Pedroso de Lima).
}

ASFV particles, which present an icosahedral shape and an average diameter of $200 \mathrm{~nm}$ (Almeida et al., 1967; Breese and DeBoer, 1966; Carrascosa et al., 1984; Schloer, 1985), are composed of several concentric domains: an internal core consisting of a central DNA-containing nucleoid coated by a thick protein layer similar to a matrix domain, referred to as core shell; an inner lipid envelope, derived from the endoplasmic reticulum; and an icosahedral protein capsid (Andres et al., 1997, 1998). The extracellular viral particles contain an additional lipid envelope acquired in the process of budding through the plasma membrane (Breese and DeBoer, 1966). ASFV resembles the poxviruses in genome structure and gene expression strategy (Baroudy et al., 1983; Gonzalez et al., 1986; Moss, 1996; Salas et al., 1999), while being morphologically similar to the iridoviruses (Carrascosa et al., 1984; Goorha and Granoff, 1994), and therefore has been described as a missing evolutionary link between the Poxviridae and Iridoviridae (Salas et al., 1999). 
Although initially it was reported that ASFV multiplied exclusively in the cytoplasm of infected cells, evidence demonstrating that the virus does not grow and no viral DNA synthesis occurs in enucleated Vero cells (Ortin and Vinuela, 1977) suggested the involvement of the host cell nucleus in the process of ASFV genome replication. Results from autoradiography studies of ASFV-infected macrophages, as well as from in situ hybridization experiments performed in both macrophages and Vero cells, have demonstrated that virus-specific DNA sequences are localized inside the nucleus of cells at early times of infection, suggesting that viral DNA synthesis is initiated inside the nucleus, being followed by a longer replication stage that occurs in the cytoplasm (Garcia-Beato et al., 1992; Rojo et al., 1999).

Analysis of the replicative intermediates that are synthesized in the nucleus and cytoplasm of ASFV-infected cells has shown that small DNA fragments are synthesized in the nucleus at early times of viral DNA replication, whereas large molecules are synthesized in the cytoplasm at later times. Based on these observations, a de novo start mechanism of ASFV DNA replication, similar to the model of Baroudy et al. (1983) for the vaccinia virus DNA replication, has been proposed (Rojo et al., 1999).

Although ASFV DNA replication has been described to occur in two distinct subcellular compartments (nucleus and cytoplasm), no information exists on the mechanisms by which the ASFV DNA is transported between the nucleus and the cytoplasm of infected cells. Most likely, this transport occurs through the nuclear pore complex and is mediated by cellular and/or viral proteins, similarly to what has been described for other viruses (reviewed in Whittaker and Helenius, 1998; Whittaker et al., 2000; Whittaker, 2003; Greber and Fassati, 2003; Greber and Fornerod, 2004; Bukrinsky, 2005). In this context, the identification and characterization of ASFV proteins that exhibit nucleo-cytoplasmic transport activity are of particular interest.

Recently, we have described p37 protein, a product of the proteolytic processing of the polyprotein pp220 (Simon-Mateo et al., 1993), as the first nucleo-cytoplasmic shuttling protein encoded by ASFV (Eulalio et al., 2004). Additionally, we have demonstrated that the nuclear export of ASFV p37 protein in Vero cells, when studied isolated from the virus infection, is a very efficient process, which occurs both by the CRM1dependent and a CRM1-independent nuclear export pathways (Eulalio et al., 2006).

In the present study we show that the ASFV $\mathrm{p} 37$ protein of the incoming virus is localized in distinct nuclear regions of Vero cells at early stages of infection, whereas at later times postinfection the newly synthesized protein is localized exclusively in the cytoplasm, concentrated in the viral factories. Additionally, we demonstrate that inhibition of the CRM1 nuclear export pathway has no effect on the subcellular localization of p37 protein in ASFV-infected cells, as well as on the kinetics of viral replication. Finally, immunofluorescence and in situ hybridization experiments on the subcellular localization of p37 protein and ASFV DNA demonstrate a clear co-localization of p37 and DNA in nuclear foci at early times post-infection, as well as in the viral factories at late stages of infection. Overall, these results are consistent with a role for $\mathrm{p} 37$ protein in the nuclear transport of viral DNA during the viral replication cycle.

\section{Materials and methods}

\subsection{Cell culture and virus}

Vero cells were grown and maintained in Dulbecco's modified Eagle's medium-high glucose (DMEM-HG) (Sigma), supplemented with $10 \%(\mathrm{v} / \mathrm{v})$ heat-inactivated fetal bovine serum (FBS) (Biochrom KG) and with $100 \mathrm{U}$ of penicillin and $100 \mu \mathrm{g}$ of streptomycin (Sigma) per ml, in a $5 \% \mathrm{CO}_{2}$ humidified atmosphere, at $37^{\circ} \mathrm{C}$.

The ASFV BA71V strain, adapted to grow in Vero cells, has already been described (Enjuanes et al., 1976). Highly purified BA71V virus was obtained by Percoll equilibrium centrifugation (Carrascosa et al., 1985).

\subsection{Anti-p37 antibody}

The antibody against ASFV p37 protein used in the immunofluorescence studies was obtained from rabbits immunized with the synthetic peptide NLTHNKQEFQSYEENY, which corresponds to amino acids 39-54 of this protein. The antibody was purified by affinity chromatography (Eurogentec), and the specificity of the purified antibody was characterized by Western-blot and immunofluorescence analysis in ASFVinfected Vero cells.

\subsection{Western-blot analysis}

For Western-blot analysis, Vero cells were either mockinfected or infected with ASFV, and $24 \mathrm{~h}$ post-infection the cells were lysed in electrophoresis sample buffer $(62.5 \mathrm{mM}$ Tris-HCl, pH 6.8, 2\% SDS, 5\% $\beta$-mercaptoethanol, $10 \%$ glycerol, and $0.01 \%$ bromophenol blue). After brief sonication, the samples were heated to $95^{\circ} \mathrm{C}$ for $10 \mathrm{~min}$. Equivalent protein amounts of the cell lysates, as well as $1 \mu \mathrm{g}$ of purified ASFV particles, were separated on a 10\% SDS-PAGE gel and the resolved proteins were transferred onto nitrocellulose membranes (Amersham Biosciences). The blot was blocked with $5 \%(\mathrm{w} / \mathrm{v})$ dry milk in Tris-buffered saline plus Tween (TBST), and then incubated with the anti-p37 antibody or with the mouse monoclonal anti-actin antibody (Sigma) in TBST containing 1\% dry milk powder. After washing three times with TBST, the membrane was incubated with peroxidase-conjugated secondary antibodies (Amersham Life Sciences). The blots were revealed by electrochemiluminescence (ECL System, Amersham Life Sciences), according to the manufacturer's instructions.

\subsection{Immunofluorescence analysis}

Preconfluent cultures of Vero cells, grown in glass coverslips (13 mm diameter), were either mock-infected or infected with $\mathrm{ASFV}$, at a multiplicity of infection of $40 \mathrm{pfu} / \mathrm{cell}$ for immunofluorescence analysis at $0,2,4,6$ and $8 \mathrm{hpi}$, or with $10 \mathrm{pfu} / \mathrm{cell}$ for the immunofluorescence analysis at 14 and $18 \mathrm{hpi}$. To synchro- 
nize the infection process, adsorption of ASFV to the cells was performed for $2 \mathrm{~h}$ at $4{ }^{\circ} \mathrm{C}$, in DMEM-HG supplemented with $2 \%$ (v/v) FBS and $25 \mathrm{mM}$ HEPES, pH 7.4, with gentle stirring. Following adsorption, the cells were washed thoroughly with DMEM-HG supplemented with $2 \%$ (v/v) FBS, and the infection was allowed to progress at $37^{\circ} \mathrm{C}$, for the indicated times. Parallel experiments were performed in the presence of $50 \mu \mathrm{g} / \mathrm{ml}$ of cytosine arabinoside (AraC). Following the indicated incubation periods, mock-infected and ASFV-infected cells were washed with PBS and fixed with $4 \%$ paraformaldehyde for $15 \mathrm{~min}$, followed by $5 \mathrm{~min}$ incubation in methanol at $-20^{\circ} \mathrm{C}$. The cells were then permeabilized with $0.2 \%$ Triton $\mathrm{X}-100$ in PBS for 5 min at room temperature, blocked with $3 \%$ bovine serum albumin (BSA) in PBS at room temperature for $30 \mathrm{~min}$, and incubated for $1 \mathrm{~h}$ at room temperature with the anti-p37 antibody and the anti- $\alpha$-tubulin monoclonal antibody (Sigma), in blocking solution. After extensive washing with PBS, the cells were incubated for $1 \mathrm{~h}$, at room temperature, with Alexa Fluor 488 goat antirabbit IgG and Alexa Fluor 594 goat anti-mouse IgG antibodies (Molecular Probes), diluted in blocking solution. The cells were further washed and the coverslips were mounted onto glass slides using Mowiol (Calbiochem).

Fluorescence observations were performed using a BIORAD Radiance 2000 laser scanning fluorescence confocal microscope, at a $600 \times$ magnification.

\subsection{Leptomycin B treatment and CRM1 silencing experiments}

Preconfluent monolayers of Vero cells, seeded in 24 well plates (wells of $2 \mathrm{~cm}^{2}$ ) and pre-treated or not with $20 \mathrm{ng} / \mathrm{ml}$ of leptomycin B (LMB) for $1 \mathrm{~h}$, were infected with ASFV BA71V strain, at a multiplicity of infection of $10 \mathrm{pfu} / \mathrm{cell}$ (for virus titration experiments) or $40 \mathrm{pfu} / \mathrm{cell}$ (for immunofluorescence experiments) in DMEM supplemented with $2 \%$ (v/v) FBS, in the presence or not of LMB $(20 \mathrm{ng} / \mathrm{ml})$. After $2 \mathrm{~h}$ of adsorption, the inoculum was removed and the cells were washed with fresh DMEM and further incubated, in DMEM supplemented with $2 \%$ FBS containing or not LMB $(20 \mathrm{ng} / \mathrm{ml})$, for the indicated times.

The short interfering RNA duplex used for depletion of CRM1 receptor (CRM1 siRNA) (sense UGUGGUGAAUUGCUUAUACd(TT); anti-sense GUAUAAGCAAUUCACCACAd(TT)) (Lund et al., 2004), targeted to the residues 90-108 of the human CRM1 receptor, was obtained from Qiagen. Twenty-four hours prior to infection of cells with ASFV BA71V strain, preconfluent monolayers of Vero cells were transfected with the siRNAs using Lipofectamine 2000 (Invitrogen), according to the manufacturer's instructions. Reduction of the expression levels of the CRM1 receptor was confirmed by Western-blot in parallel experiments (data not shown), as described previously (Eulalio et al., 2006).

\subsection{Plaque assays}

The cells infected with ASFV BA71V strain and their culture supernatants, under the different experimental conditions, were harvested at different times post-infection, sonicated and titrated by plaque assay (Enjuanes et al., 1976).

\subsection{In situ hybridization with ASFV-specific digoxigenin-labeled DNA probes}

Total viral DNA isolated from purified virions was used as a probe to detect ASFV DNA in the in situ hybridization experiments, as previously reported (Garcia-Beato et al., 1992). The DNA probe was labeled with digoxigenin-11-dUTP, according to the manufacturer's instructions (Roche Applied Science).

The procedure for the in situ hybridization experiments using digoxigenin-labeled probes has been previously described by Garcia-Beato et al. (1992). Briefly, mock-infected and ASFVinfected Vero cells cultured in activated coverslips were fixed at distinct times post-infection with a solution of ethanol:acetic acid (3:1). After digestion with proteinase $\mathrm{K}(1 \mu \mathrm{g} / \mathrm{ml})$ for $15 \mathrm{~min}$ at $37^{\circ} \mathrm{C}$, the samples were treated with $100 \mu \mathrm{g} / \mathrm{ml}$ of RNAse A and $10 \mathrm{U} / \mathrm{ml}$ RNAse $\mathrm{T} 1$ for $30 \mathrm{~min}$ at $37^{\circ} \mathrm{C}$. The DNA was denatured by incubating the samples in a solution containing $95 \%$ freshly deionized formamide and $0.1 \times \mathrm{SSC}$ at $65^{\circ} \mathrm{C}$ for $15 \mathrm{~min}$. Prehybridization was then performed by incubation of the samples for $15 \mathrm{~min}$ at $42{ }^{\circ} \mathrm{C}$ in $45 \%$ formamide, $6 \times$ SSC, $5 \times$ Denhardt's solution and $100 \mu \mathrm{g} / \mathrm{ml}$ denatured salmon sperm DNA. For the hybridization step, the cell samples were incubated overnight in $45 \%$ formamide, $6 \times$ SSC, $5 \times$ Denhardt's solution and $10 \%$ dextran sulphate containing $10 \mathrm{ng}$ of denaturated ASFV DNA probe under siliconized coverslips, at $42{ }^{\circ} \mathrm{C}$ in a humidified chamber. Following the hybridization step, the samples were washed thoroughly with $2 \times \mathrm{SSC}$ at room temperature, followed by permeabilization with $0.2 \%$ Triton $\mathrm{X}-100$ in PBS. After extensive washing with PBS, the samples were then incubated with $50 \mu \mathrm{g} / \mathrm{ml}$ sheep anti-digoxigenin Fab fragments conjugated with fluorescein (Roche Applied Science) in PBS containing $0.5 \%$ BSA and $1 \%$ blocking reagent (Roche Applied Science), for $1 \mathrm{~h}$ at room temperature. The samples were then processed for immunofluorescence as described above, and labeled with anti-p37 and anti- $\alpha$-tubulin antibodies, recognized, respectively by Alexa Fluor 594 goat anti-rabbit IgG and Alexa Fluor 647 goat anti-mouse IgG (Molecular Probes).

\section{Results}

\subsection{ASFV p37 protein is localized in distinct subcellular compartments during viral infection}

To examine the subcellular localization of $\mathrm{p} 37$ protein during the process of infection by ASFV, immunofluorescence studies were performed using a new antibody raised against ASFV p37 protein (amino acids 39 to 54; Section 2.2). The specificity of this antibody was demonstrated by Western-blot analysis, which showed that the anti-p37 antibody does not recognize any protein in the extract from non-infected cells (mock cells), whereas in the extract from ASFV-infected cells the antibody recognizes ASFV p37 protein (Fig. 1). As expected, the antibody also recognizes the precursors of $\mathrm{p} 37$ protein, namely the immediate 


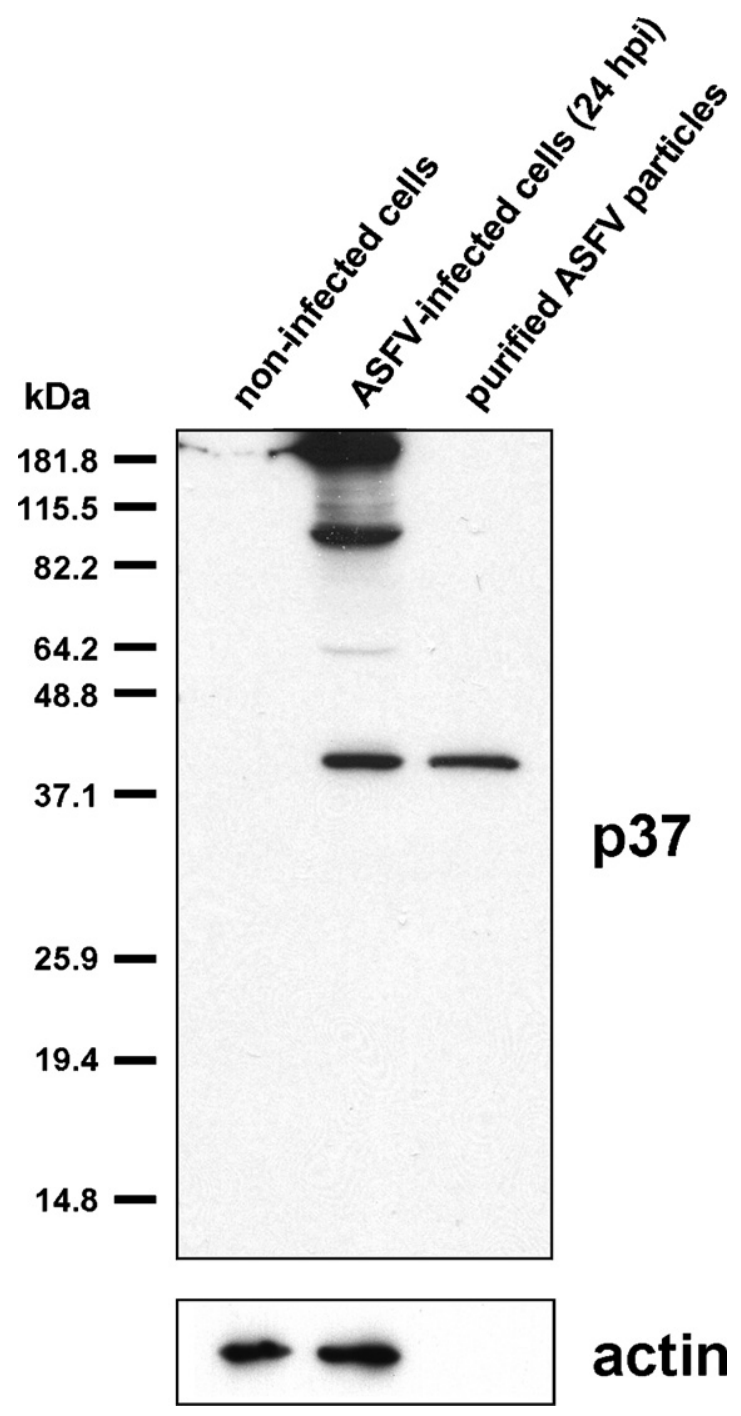

Fig. 1. Western-blot analysis of the specificity of the anti-p37 antibody. Equivalent protein amounts of lysates of mock-infected or ASFV-infected Vero cells, as well as $1 \mu \mathrm{g}$ of purified ASFV particles were separated by SDS-PAGE and analyzed by Western-blot using an anti-p37 antibody obtained as described in Section 2.2. Reprobing with anti-actin antibody was performed as control.

polyprotein precursor pp55 (which originates p14 and p37), the polyprotein precursor pp90 (which originates pp55 and p34), and finally the pp220 polyprotein. In purified virus particles the antibody only recognized the p37 protein, in agreement with observations demonstrating that only the final and correctly processed products of polyprotein precursor pp220 are incorporated into virus particles (Simon-Mateo et al., 1993) (Fig. 1).

As shown in Fig. 2, p37 protein was not detected by immunofluorescence in ASFV-infected Vero cells at very early times post-infection ( 0 and $2 \mathrm{hpi})$, most likely because the antibody has no access to the protein localized in the inner core shell domain of the incoming virus particles (Andres et al., 1997, 2002a,b). The p37 protein was first detected at $4 \mathrm{hpi}$, being found both in the cytoplasm and nucleus, accumulating in specific nuclear regions. At $6 \mathrm{hpi}, \mathrm{p} 37$ protein accumulation in distinct regions inside the nucleus of infected Vero cells was considerably more pronounced than at earlier times, although the protein continued to be detected throughout the cytoplasm of the infected cells.

Interestingly, at later times of the infection cycle, namely $8 \mathrm{hpi}$, the nuclear signal of $\mathrm{p} 37$ protein was considerably weaker, the protein being localized mainly in the cytoplasm of the infected cells in a diffuse pattern (Fig. 2).

At 14 and $18 \mathrm{hpi}$, the $\mathrm{p} 37$ protein was concentrated in the viral factories, although fluorescence was also detected throughout the cytoplasm (Fig. 2). The signal detected at the viral factory most likely corresponds to the newly synthesized p37 protein and its precursor, which are induced at these late times of the infection cycle and are known to localize at the virus assembly sites (Andres et al., 1997).

Representative images from immunofluorescence experiments in mock-infected cells (at $14 \mathrm{hpi}$ ), which were performed as a control to demonstrate the specificity of the anti-p37 antibody, are shown for comparison. The subcellular localization of $\alpha$-tubulin was simultaneously determined to facilitate the analysis of the localization of the p37 protein.

The presence of the $\mathrm{p} 37$ protein inside the nucleus at the initial stages of the infection cycle, followed by its disappearance from this compartment at later times, may suggest a role of this protein related to the nuclear phase of viral DNA replication.

\subsection{ASFV p37 protein detected at early times of infection originates from infecting ASFV particles, rather than from de novo protein synthesis}

It is well documented that ASFV pp220 polyprotein and, consequently all the products of its proteolytic processing including p37 protein, are late viral proteins (Andres et al., 1993; SimonMateo et al., 1993), whose synthesis is initiated only after viral DNA replication. This finding suggests per se that the p37 protein that is detectable by immunofluorescence at early times of infection is that derived from incoming ASFV particles, which are responsible for cell infection.

To clarify this issue, immunofluorescence experiments on the subcellular localization of $\mathrm{p} 37$ protein were performed in ASFVinfected Vero cells following treatment with AraC, a drug that blocks the activity of cellular and viral DNA polymerases, thus inhibiting viral DNA replication and consequently the synthesis of late ASFV proteins, including the polyprotein pp220 (Andres et al., 1993).

In ASFV-infected and AraC-treated cells, p37 protein presented the same subcellular localization as in infected and non-treated cells, being localized in specific nuclear regions and in the cytoplasm at $6 \mathrm{hpi}$, and absent from the nucleus at intermediate times of infection (8 hpi) (compare Fig. 3 with Fig. 2). As expected, no $\mathrm{p} 37$ protein was detected at late times post-infection (14 hpi) in ASFV-infected and AraC-treated cells (Fig. 3), since cell treatment with AraC indirectly impairs the synthesis of late ASFV proteins. These results demonstrate that the $\mathrm{p} 37$ protein detected by immunofluorescence at early and intermediate times of infection ( 6 and $8 \mathrm{hpi}$ ) is the protein that entered into Vero cells incorporated in the infecting virus particles, rather than newly synthesized protein. 

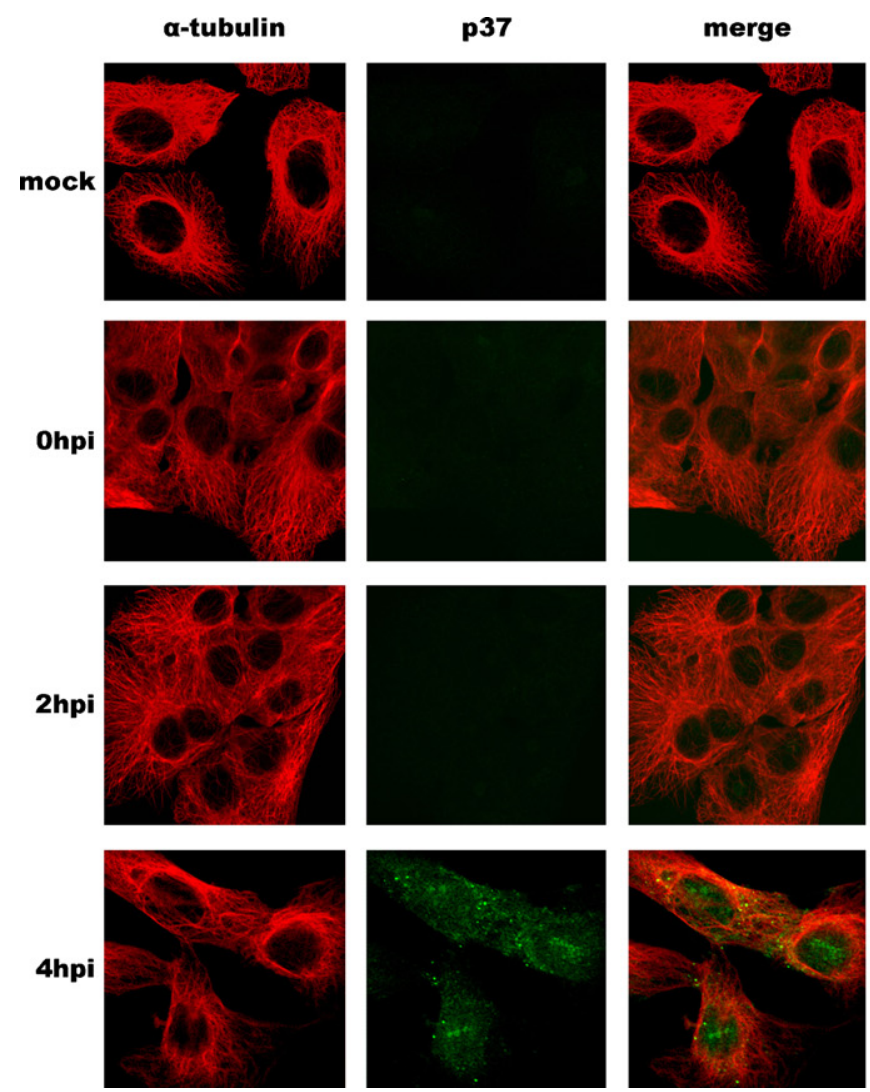

a-tubulin
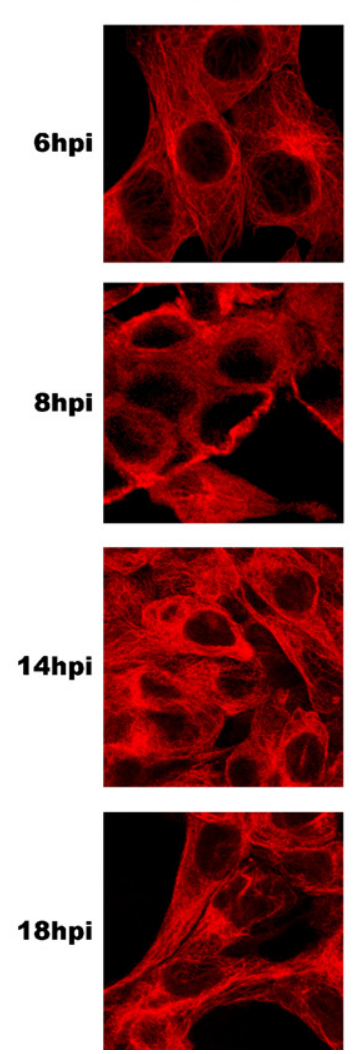

p37
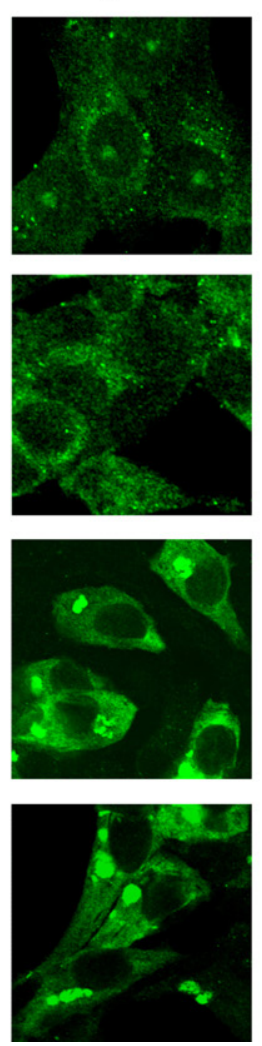

merge
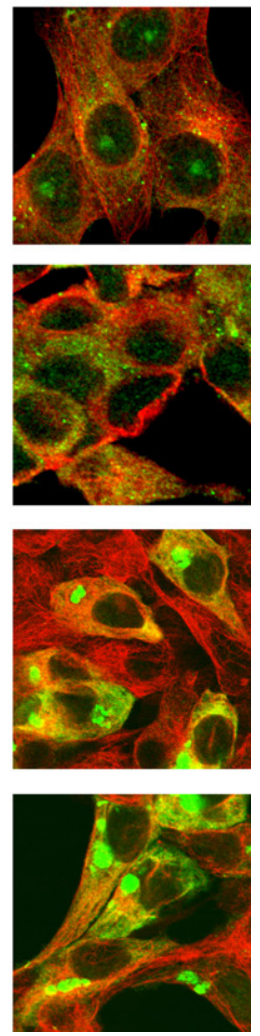

Fig. 2. Subcellular localization of p37 protein in ASFV-infected cells, at different times post-infection. Vero cells infected with ASFV BA71V strain were fixed and processed for immunofluorescence analysis at the indicated times post-infection. Samples were incubated with antibodies against p37 protein and $\alpha$-tubulin, followed by incubation with secondary antibodies conjugated to Alexa 488 and 594, respectively. Experiments were performed at least three times and representative confocal microscopy images are shown.

\subsection{The subcellular localization of p37 protein in ASFV-infected cells is not affected by inhibition of the CRM1-dependent nuclear export pathway}

We have previously reported that the nuclear export of p37 protein in Vero cells is a very efficient process that occurs through two distinct pathways: the CRM1-dependent and an unidentified CRM1-independent pathway (Eulalio et al., 2006). Moreover, we have demonstrated that the nuclear export of full-length p37 protein is partially inhibited by both specific depletion of the CRM1 receptor by RNA interference and leptomycin $\mathrm{B}$, a metabolite that potently and specifically blocks the CRM1-dependent nuclear export pathway by binding directly to a cysteine residue of the CRM1 receptor (Eulalio et al., 2006).

In this context, aiming at investigating the contribution of the CRM1-dependent pathway to the nuclear export of $\mathrm{p} 37$ protein in ASFV-infected Vero cells, the subcellular localization of p37 protein was analysed at different times post-infection, in cells in which this nuclear export pathway was specifically inhibited either by leptomycin B or siRNAs targeted to the CRM1 receptor.

At early times of infection (4 and $6 \mathrm{hpi}$ ), there was no change in p37 protein localization in infected cells that were either treated with LMB or transfected with siRNAs targeted to the CRM1 receptor, the protein being located in the nucleus and cytoplasm similarly to what was observed in non-treated cells (compare Fig. 4 with Fig. 2). Accordingly, at intermediate and late times of infection ( 8 and $14 \mathrm{hpi}$ ) no nuclear accumulation of $\mathrm{p} 37$ protein was detected upon inhibition of the CRM1dependent pathway by either of the two referred experimental approaches, the protein remaining localized exclusively in the cytoplasm of the ASFV-infected cells (Fig. 4).

Overall, these results clearly show that, in ASFV-infected cells, no significant differences are observed in the subcellular localization of p37 protein upon inhibition of the CRM1mediated nuclear export pathway.

\subsection{Inhibition of the CRMI-mediated nuclear export pathway has no effect on the kinetics of ASFV replication in Vero cells}

Although no significant differences in the subcellular localization of $\mathrm{p} 37$ protein were observed in ASFV-infected cells upon inhibition of the CRM1-dependent nuclear export pathway, experiments were performed to evaluate the relevance of this pathway to the kinetics of viral replication. For this purpose, the growth of the ASFV BA71V strain, which was used throughout this study, was examined in cells in which the CRM1dependent pathway was inhibited either by treatment with LMB or depletion of the CRM1 receptor using a specific siRNA, and compared to that observed in non-treated cells. 


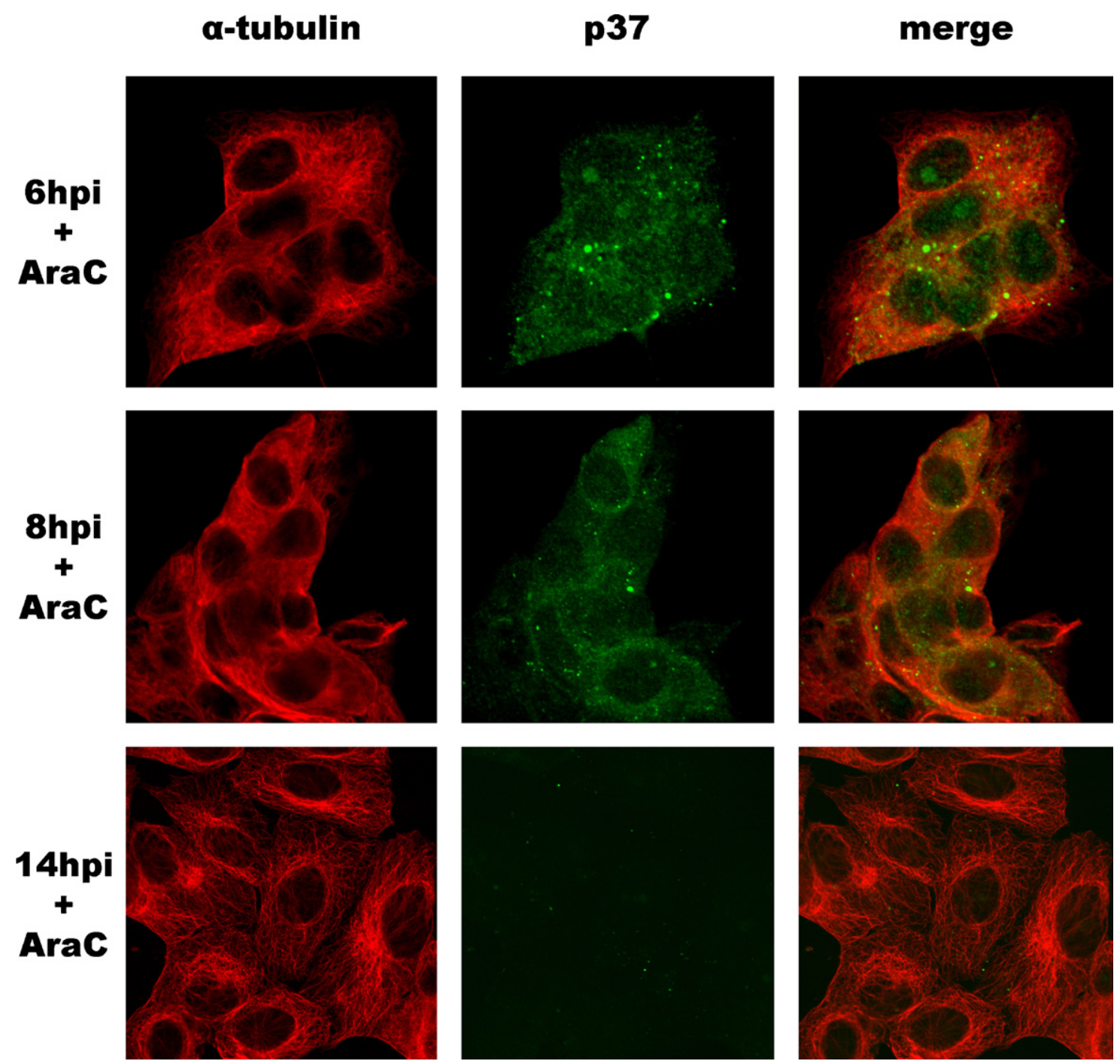

Fig. 3. Subcellular localization of $\mathrm{p} 37$ protein in ASFV-infected cells following treatment with AraC. Vero cells were infected with ASFV BA71V strain and incubated for the indicated times in the presence of $50 \mu \mathrm{g} / \mathrm{ml}$ of AraC, a drug that inhibits viral DNA replication and consequently the synthesis of late ASFV proteins. Samples were incubated with antibodies against p37 protein and $\alpha$-tubulin, followed by incubation with secondary antibodies conjugated to Alexa 488 and 594 , respectively. Experiments were performed at least three times and representative confocal microscopy images are shown (magnification $600 \times$ ).

As can be inferred from the one-step growth curves obtained under the different experimental conditions, no significant differences were observed in the yield of virus produced in the cells in which the CRM1-mediated nuclear export pathway was inhibited, when compared to that obtained in control (non-treated) cells infected with the BA71V virus (Fig. 5).

Overall, these results demonstrate that, independently of the strategy used to inhibit the nuclear export mediated by the CRM1 receptor (LMB treatment or RNA interference), the inhibition of this nuclear export pathway has no significant effect on the kinetics of ASFV replication in Vero cells.

\section{5. p37 protein and ASFV DNA co-localize in the nucleus of infected Vero cells at early times post-infection}

Previous studies have shown that specific ASFV DNA sequences are present in the nucleus of infected Vero cells at early times of infection, accumulating in dense foci localized in the vicinity of the nuclear membrane, whereas at later stages of infection, the viral DNA is localized exclusively in the cytoplasm, concentrated in the viral factories (Garcia-Beato et al., 1992).

To determine whether, at early stages of viral infection, the specific nuclear regions of $\mathrm{p} 37$ protein accumulation correspond to the foci where the viral DNA is present, the subcellular localization of p37 protein and viral DNA was simultaneously determined by immunofluorescence and in situ hybridization experiments in Vero cells. To facilitate the analysis, immunofluorescence labelling of the cytoplasmic marker $\alpha$-tubulin was also performed.

As can be observed in Fig. 6, in situ hybridization experiments showed that, at $6 \mathrm{hpi}$, the ASFV DNA is concentrated in distinct foci inside the nucleus. As previously shown, at $6 \mathrm{hpi}$, p37 protein is also localized in distinct nuclear regions. Remarkably, the merged confocal images demonstrate that $\mathrm{p} 37$ protein and the ASFV DNA are co-localized in these distinct foci. Further- 
+LMB
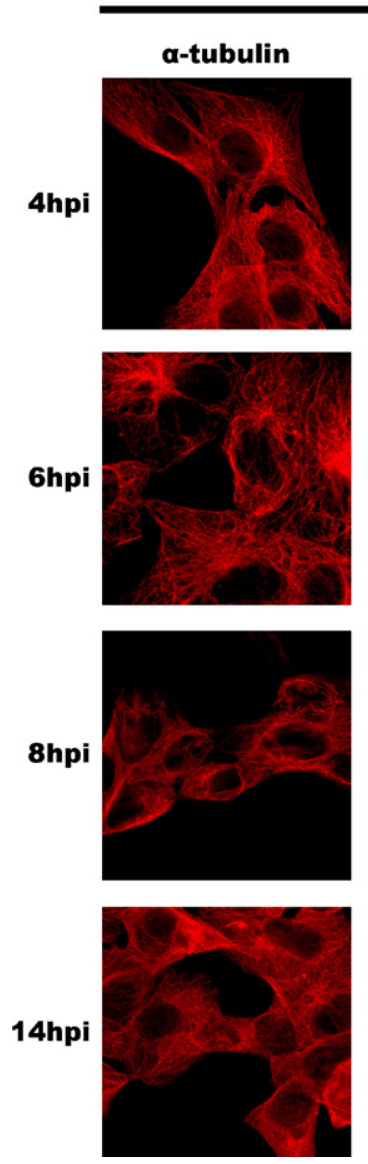
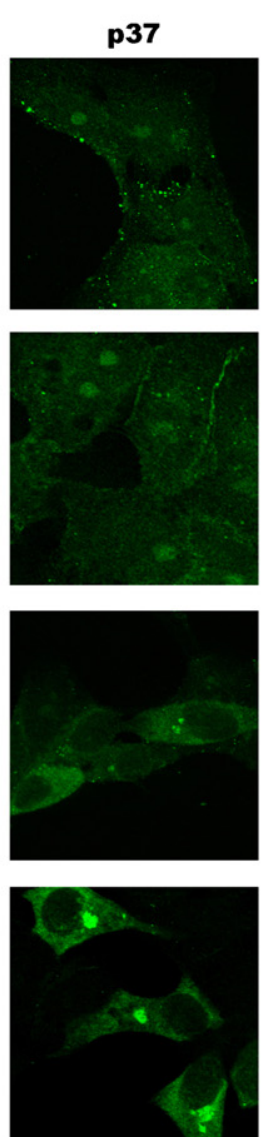
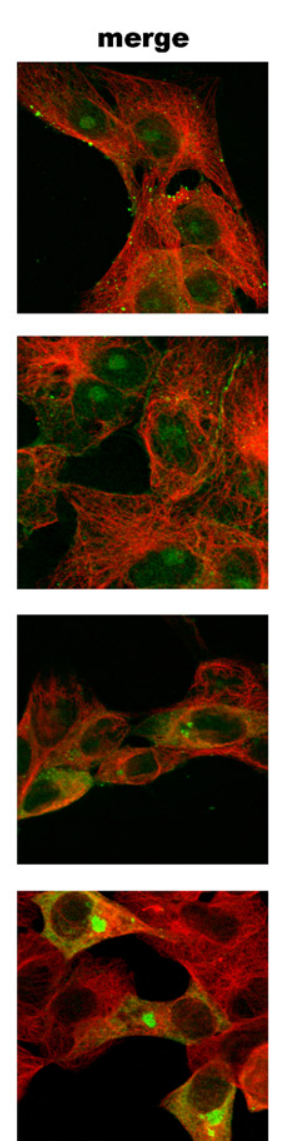

+CRM1 SIRNA
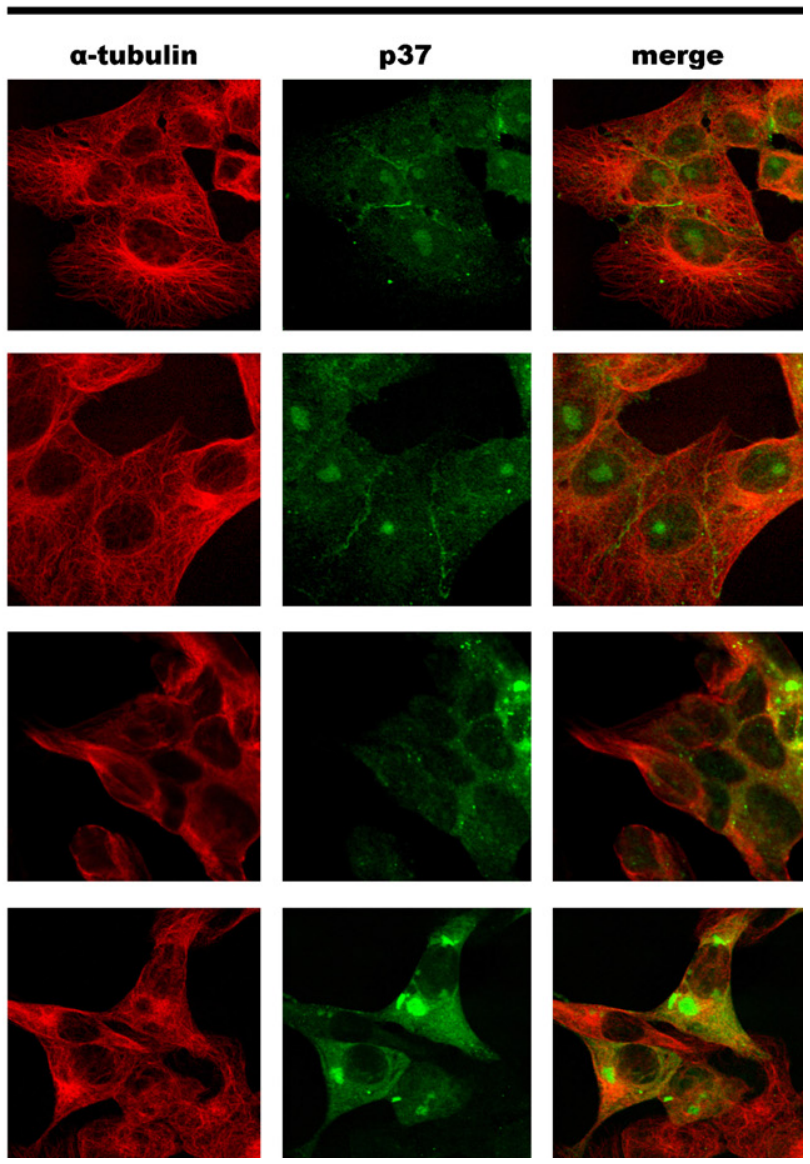

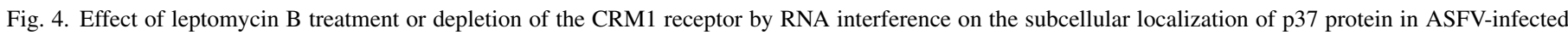

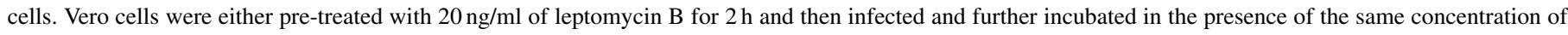

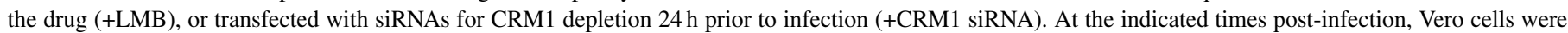

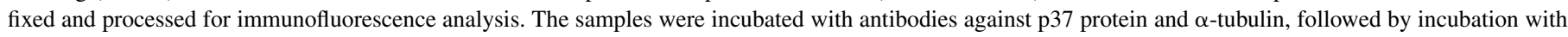

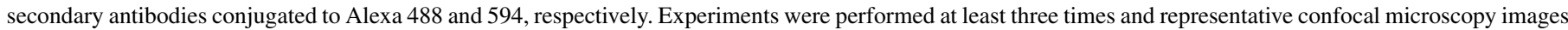
are shown (magnification $600 \times$ ).

more, labelling of the cytoplasm with anti- $\alpha$-tubulin antibody undoubtedly confirms that those foci are localized inside the nucleus.

At intermediate times post-infection ( $8 \mathrm{hpi}$ ), both the ASFV DNA and p37 protein are absent from the nucleus, being detected exclusively in the cytoplasm of the infected cells, concentrated in regions that are most likely precursors of the viral factories (Fig. 6). At later times (14 hpi), the overlaid confocal images show an evident co-localization of the ASFV DNA and p37 protein, or its polyprotein precursors, in the viral factories (Fig. 6).

Control experiments with mock-infected cells show a low non-specific background staining of the cytoplasm, but not inside the nucleus, confirming the specificity of the hybridization signal detected in ASFV-infected cells.

It should be noted that due to the experimental procedure used in the in situ hybridization studies, which involves treatment of cells with proteinase $\mathrm{K}$ to allow the accessibility of the digoxigenin-labeled DNA probe to the viral DNA, the signal corresponding to $\mathrm{p} 37$ protein was weaker than that obtained in the immunofluorescence experiments described in the previous sections.
The co-localization of the viral DNA and p37 protein in distinct nuclear regions at the initial stages of the infection cycle and their simultaneous disappearance from the nucleus at later times are consistent with the involvement of $\mathrm{p} 37$ protein in the nucleo-cytoplasmic transport of the ASFV DNA.

\section{Discussion}

Many viruses have a nuclear replication stage or replicate exclusively inside the nucleus of their host cells, and therefore the nucleo-cytoplasmic transport of viral components plays a central role in their replication cycle. With the exception of a few viruses, the transport across the nuclear envelope during viral replication occurs through the nuclear pore complex and exploits the host cellular machinery, in particular the nuclear import and export receptors and transport factors (Whittaker and Helenius, 1998; Whittaker et al., 2000; Whittaker, 2003; Greber and Fassati, 2003; Greber and Fornerod, 2004; Bukrinsky, 2005).

In this context, the existence of an initial phase of ASFV genome replication inside the nucleus, followed by a longer replication stage in the cytoplasm (Garcia-Beato et al., 1992; 


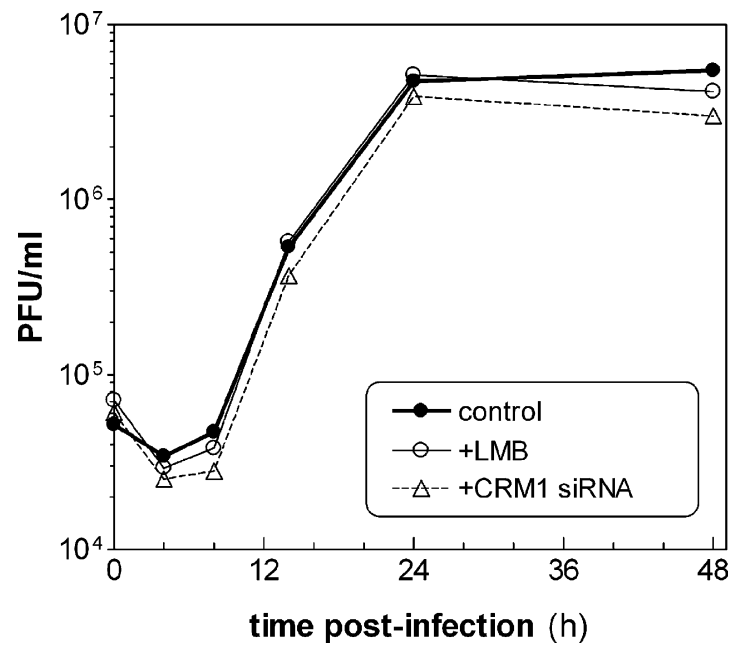

Fig. 5. One-step growth curves of BA71V virus produced in the presence of leptomycin B or in CRM1-depleted Vero cells. Infection with ASFV BA71V strain (10 pfu/cell) was performed in: (i) non-treated Vero cells (control); (ii) Vero cells treated with $20 \mathrm{ng} / \mathrm{ml}$ of leptomycin B (+LMB); or (iii) Vero cells transfected with siRNAs for CRM1 depletion $24 \mathrm{~h}$ prior to infection (+CRM1 siRNA). At different times post-infection, the samples were collected and titrated by plaque assay on Vero cells.

Rojo et al., 1999), strongly suggests that some viral proteins, together with the viral DNA, are actively transported between the nucleus and cytoplasm of host cells.

Following previous studies in our laboratory demonstrating that $\mathrm{p} 37$ protein, when studied isolated from the virus infection, is actively transported between the nucleus and the cytoplasm of Vero cells (Eulalio et al., 2004), the evaluation of the nucleocytoplasmic transport activity of this protein during ASFV infection was considered pertinent.

The results presented here show that $\mathrm{p} 37$ protein is present in distinct subcellular compartments at different times of the infection cycle. At early times post-infection, the protein is present in the cytoplasm and nucleus of Vero cells, particularly concentrated in distinct nuclear regions, whereas at later times the p37 protein is absent from the nucleus, being detected exclusively in the cytoplasm of virus-infected cells. Results obtained from experiments performed in ASFV-infected cells in which late gene expression was inhibited demonstrate that the p37 protein detected at early times post-infection is the structural p37 protein of the incoming virus particles, which is thus able to enter the nucleus at these times.

On the other hand, the protein detected in viral factories at late times post-infection corresponds to newly synthesized p37 protein, and/or its polyprotein precursors. Since p37 protein is localized in the viral core shell, a matrix-like domain that surrounds the DNA-containing nucleoid of the virus (Andres et al., 1997, 2002a,b), a likely explanation for the fact that p37 protein has not been detected at very early times post-infection, namely at 0 and $2 \mathrm{hpi}$, is that the anti-p37 antibody access to p37 protein in the viral particle has been hampered, even following cell permeabilization. According to this hypothesis, the antibody would only have access to $\mathrm{p} 37$ protein after the disassembly of virus particles, particularly the capsid breakdown, which would explain the detection of the protein only at $4 \mathrm{~h}$ post-infection.
The observation that, in contrast to $\mathrm{p} 37$ protein, the $\mathrm{p} 72 \mathrm{ASFV}$ protein is detected by immunofluorescence at very early times post-infection (data not shown) also supports this hypothesis since p72 protein is the major capsid component.

The fate of the nuclear p37 protein is not clear at present. Our previous results have demonstrated that the nuclear export of p37 protein is very efficient, comparable to the nuclear export mediated by the nuclear export signals (NES) present in HIV-1 Rev protein. Furthermore, it was shown that p37 protein nuclear export is mediated by three NESs and occurs through two distinct nuclear export pathways, the CRM1-dependent and an unidentified CRM1-independent pathway (Eulalio et al., 2006). Since the nuclear export of p37 protein mediated by a CRM1independent pathway has a strength comparable to that mediated by the CRM1-dependent pathway (Eulalio et al., 2006), it is possible that, in the context of full-length p37 protein and particularly during the ASFV infection, the protein will be able to exit the nucleus even when the CRM1-dependent route is inhibited, by using a CRMI-independent pathway. This would explain the results obtained from the immunofluorescence experiments with cells infected in the presence of leptomycin B, in which the subcellular localization of $\mathrm{p} 37$ is the same as that observed in non-treated cells.

Given the large size of the ASFV genome, a linear doublestranded DNA molecule of 170-190 kbp depending on the virus isolate (Blasco et al., 1989), it is clear that the viral DNA can only enter into the nucleus by an active transport process. Likewise, the exit of the replicating viral DNA, as well as of the replicative intermediates, from the nucleus is most likely a very complex process. By analogy to other viruses, it can be speculated that the ASFV DNA is transported into the nucleus of infected cells as a complex with viral, and perhaps, cellular proteins, rather than in an isolated form.

Previous results from in situ hybridization experiments in ASFV-infected Vero cells have demonstrated that, at early times of infection, the viral DNA is localized in distinct nuclear regions (Garcia-Beato et al., 1992). In this context, the observation reported in the present study of a similar pattern of localization for $\mathrm{p} 37$ protein prompted us to perform studies aimed at simultaneously determine the subcellular localization of the p37 protein and viral DNA. Remarkably, our results show a perfect co-localization of the ASFV DNA and p37 protein in specific nuclear regions, at early times post-infection, whereas at later times of infection both of them are localized in the cytoplasm, concentrated in the viral factories.

These observations indicate that during the early nuclear stage of ASFV genome replication at least one viral protein is present in the subnuclear region where the viral DNA is localized, reinforcing the importance of investigating the early steps of the viral replication cycle.

Presently, the requirement of the nucleus for ASFV DNA replication is not clear. Although it has been demonstrated that the virus encodes most of the proteins required for its genome replication, namely a DNA polymerase (Rodriguez et al., 1993), a homologue of the proliferating cell nuclear antigen (PCNA) (Yanez et al., 1995), a DNA ligase (Yanez and Vinuela, 1993), a type II topoisomerase (Baylis et al., 1992; Garcia-Beato et al., 


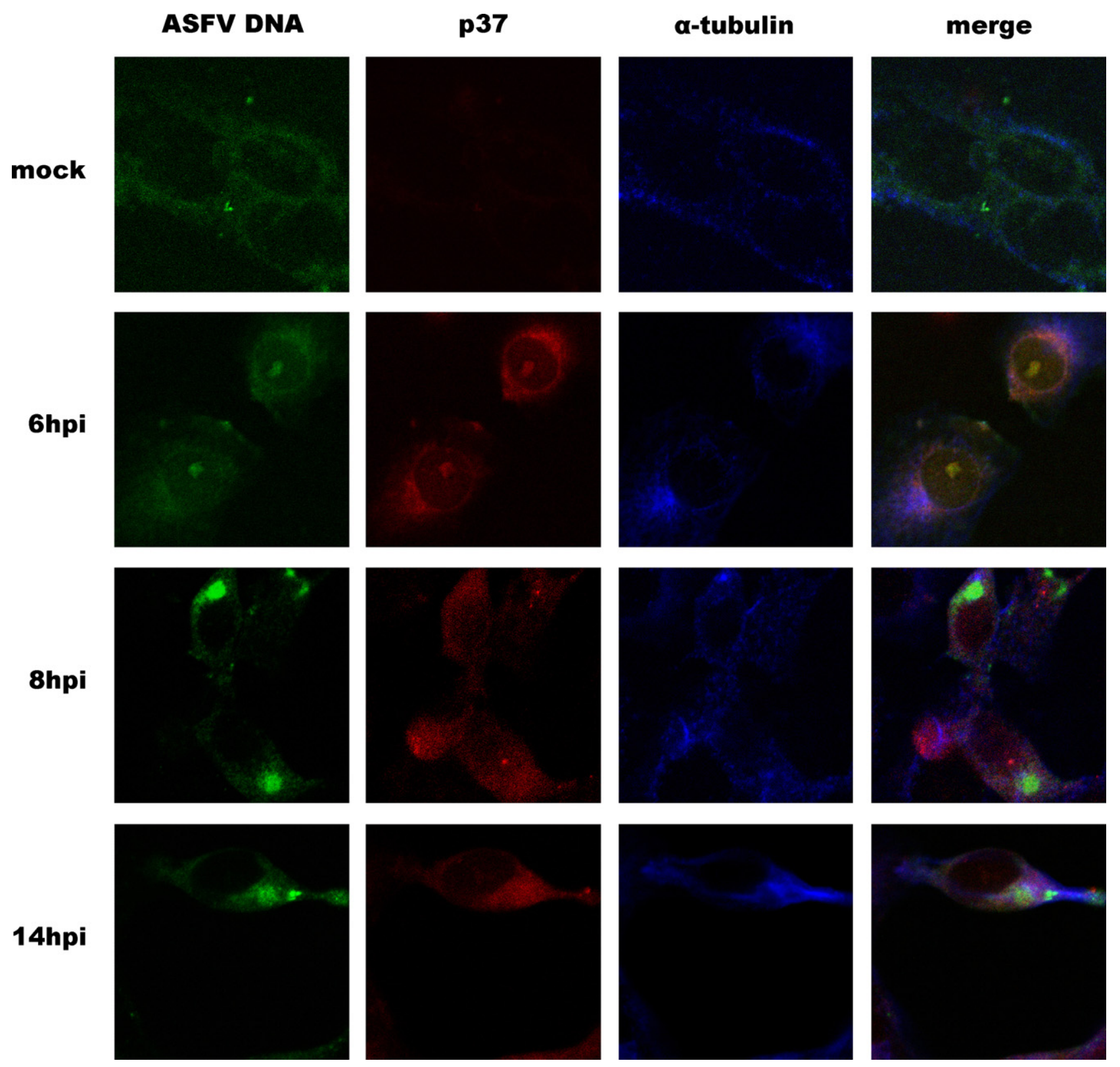

Fig. 6. Simultaneous evaluation of the subcellular localization of ASFV DNA and p37 protein in ASFV-infected cells, at different times post-infection. Mock-infected and ASFV-infected Vero cells were fixed and processed for simultaneous in situ hybridization and immunofluorescence analysis, at the indicated times post-infection. For the in situ hybridization experiments, the digoxigenin-labelled ASFV DNA probe was detected with an anti-digoxigenin antibody conjugated with fluorescein. The cell samples were also incubated with the anti-p37 protein and anti- $\alpha$-tubulin antibodies, which were detected with secondary antibodies conjugated to Alexa 488 and 647, respectively. Experiments were performed at least three times and representative confocal microscopy images are shown (magnification $600 \times$ ).

1992) and several DNA helicases (Baylis et al., 1993; Yanez et al., 1993, 1995), the existence of a nuclear stage of replication has been attributed to a requirement for cellular proteins in the initiation of the viral DNA synthesis. Supporting this hypothesis, experiments in ASFV-infected cells treated with aphidicolin, an inhibitor of the cellular DNA polymerases $\alpha$ and $\delta$ but with no effect on the viral DNA polymerase, suggest that a cellular DNA polymerase may be involved in early steps of ASFV DNA replication (Marques and Costa, 1992).

The results described in the present report showing that the structural p37 protein of the infecting ASFV is in close association with the viral DNA during the early steps of viral DNA replication, which occur in the nucleus, suggest that this protein accompanies the viral DNA during its transport into the nucleus, which may facilitate the entry of the virus genome into this compartment to initiate replication. This is the first viral protein identified as forming part of the nuclear foci where the viral DNA is localized. In an alternative or complementary scenario, $\mathrm{p} 37$ protein could also be involved in the export of the viral genome from the nucleus to the cytoplasm of infected cells, following the brief nuclear replication phase. Most likely, other viral proteins are also involved in this process. Among others, ASFV p14 protein, a structural ASFV protein which is also a product of the proteolytic processing of the polyprotein precur- 
sor pp220, has been demonstrated to be actively imported into the cell nucleus (Eulalio et al., 2004), thus constituting another candidate to mediate the nuclear import of the ASFV genome.

It is clear that further studies are needed to characterize in detail the mechanisms of the nucleo-cytoplasmic transport of the ASFV genome. However, our observations of the distinct nuclear and cytoplasmic localizations of $\mathrm{p} 37$ protein during the viral infection cycle, which might be explained by the previously described shuttling activity of this protein, and its co-localization within the nucleus with the viral DNA, are consistent with the involvement of $\mathrm{p} 37$ protein in the nuclear transport of the virus genome.

\section{Acknowledgements}

This work was supported by a grant from the Portuguese Foundation for Science and Technology (POCTI/ CVT/44854/2002). A. Eulálio and I. Nunes-Correia are recipients of fellowships from the Portuguese Foundation for Science and Technology. The work in the laboratory of J. Salas and M.L. Salas was supported by a grant from Ministerio de Educación y Ciencia (BFU2004-00298) and by an institutional grant from the Fundación Ramón Areces.

\section{References}

Almeida, J.D., Waterson, A.P., Plowright, W., 1967. The morphological characteristics of African swine fever virus and its resemblance to tipula iridescent virus. Arch. Gesamte Virusforsch 20, 392-396.

Andres, G., Simon-Mateo, C., Vinuela, E., 1993. Characterization of two African swine fever virus 220-kDa proteins: a precursor of the major structural protein p150 and an oligomer of phosphoprotein p32. Virology 194, 284-293.

Andres, G., Simon-Mateo, C., Vinuela, E., 1997. Assembly of African swine fever virus: role of polyprotein pp220. J. Virol. 71, 2331-2341.

Andres, G., Garcia-Escudero, R., Simon-Mateo, C., Vinuela, E., 1998. African swine fever virus is enveloped by a two-membraned collapsed cisterna derived from the endoplasmic reticulum. J. Virol. 72, 8988-9001.

Andres, G., Alejo, A., Salas, J., Salas, M.L., 2002a. African swine fever virus polyproteins pp220 and pp62 assemble into the core shell. J. Virol. 76, 12473-12482.

Andres, G., Garcia, E., Salas, M.L., Rodriguez, J.M., 2002b. Repression of African swine fever virus polyprotein pp220-encoding gene leads to the assembly of icosahedral core-less particles. J. Virol. 76, 2654-2666.

Baroudy, B.M., Venkatesan, S., Moss, B., 1983. Structure and replication of vaccinia virus telomeres. Cold Spring Harb. Symp. Quant. Biol. 47, 723729.

Baylis, S.A., Dixon, L.K., Vydelingum, S., Smith, G.L., 1992. African swine fever virus encodes a gene with extensive homology to type II DNA topoisomerases. J. Mol. Biol. 228, 1003-1010.

Baylis, S.A., Twigg, S.R., Vydelingum, S., Dixon, L.K., Smith, G.L., 1993. Three African swine fever virus genes encoding proteins with homology to putative helicases of vaccinia virus. J. Gen. Virol. 74, 1969-1974.

Blasco, R., Aguero, M., Almendral, J.M., Vinuela, E., 1989. Variable and constant regions in African swine fever virus DNA. Virology 168, 330-338.

Breese, J., DeBoer, C.J., 1966. Electron microscope observations of African swine fever virus in tissue culture cells. Virology 28, 420-428.

Bukrinsky, M., 2005. Nuclear import and export of mammalian viruses. In: Tzfira, T., Citovsky, V. (Eds.), Nuclear Import and Export in Plants and Animals. Landes Bioscience/Eurekah. com and Kluwer Academic/Plenum Publishers, Georgetown, TX and New York, NY, pp. 175-186.

Carrascosa, A.L., del, V.M., Santaren, J.F., Vinuela, E., 1985. Purification and properties of African swine fever virus. J. Virol. 54, 337-344.
Carrascosa, J.L., Carazo, J.M., Carrascosa, A.L., Garcia, N., Santisteban, A., Vinuela, E., 1984. General morphology and capsid fine structure of African swine fever virus particles. Virology 132, 160-172.

Dixon, L.K., Escribano, J.M., Martins, C., Rock, D.L., Salas, M.L., Wilkinson, P.J., 2005. Asfarviridae. In: Fauquet, C.M., Mayo, M.A., Maniloff, J., Desselberger, U., Ball, L.A. (Eds.), Virus Taxonomy, VIIIth Report of the ICTV. Elsevier/Academic Press, London, pp. 135-143.

Enjuanes, L., Carrascosa, A.L., Moreno, M.A., Vinuela, E., 1976. Titration of African swine fever (ASF) virus. J. Gen. Virol. 32, 471-477.

Eulalio, A., Nunes-Correia, I., Carvalho, A.L., Faro, C., Citovsky, V., Simoes, S., Pedroso de Lima, M.C., 2004. Two African swine fever virus proteins derived from a common precursor exhibit different nucleocytoplasmic transport activities. J. Virol. 78, 9731-9739.

Eulalio, A., Nunes-Correia, I., Carvalho, A.L., Faro, C., Citovsky, V., Salas, J., Salas, M.L., Simoes, S., Pedroso de Lima, M.C., 2006. Nuclear export of African swine fever virus $\mathrm{p} 37$ protein occurs through two distinct pathways and is mediated by three independent signals. J. Virol. 80, 13931404.

Garcia-Beato, R., Salas, M.L., Vinuela, E., Salas, J., 1992. Role of the host cell nucleus in the replication of African swine fever virus DNA. Virology 188, 637-649.

Gonzalez, A., Talavera, A., Almendral, J.M., Vinuela, E., 1986. Hairpin loop structure of African swine fever virus DNA. Nucl. Acids Res. 14, 6835-6844.

Goorha, R., Granoff, A., 1994. Frog virus 3. In: Webster, R.G., Granoff, A. (Eds.), Encyclopedia of Virology. Academic Press, London, pp. 503-508.

Greber, U.F., Fassati, A., 2003. Nuclear import of viral DNA genomes. Traffic 4, 136-143.

Greber, U.F., Fornerod, M., 2004. Nuclear import in viral infections. Curr. Top. Microbiol. Immunol. 285, 109-138.

Lund, E., Guttinger, S., Calado, A., Dahlberg, J.E., Kutay, U., 2004. Nuclear export of microRNA precursors. Science 303, 95-98.

Marques, M.I., Costa, J.V., 1992. African swine fever virus-induced DNA polymerase is resistant to aphidicolin. Virology 191, 498-501.

Moss, B., 1996. Poxviridae: the viruses and their replication. In: Fields, B., Knipe, D.M., Howley, P.M. (Eds.), Fields Virology. Lippincott-Raven, Philadelphia, PA, pp. 2637-2672.

Ortin, J., Vinuela, E., 1977. Requirement of cell nucleus for African swine fever virus replication in Vero cells. J. Virol. 21, 902-905.

Rodriguez, J.M., Yanez, R.J., Rodriguez, J.F., Vinuela, E., Salas, M.L., 1993. The DNA polymerase-encoding gene of African swine fever virus: sequence and transcriptional analysis. Gene 136, 103-110.

Rojo, G., Garcia-Beato, R., Vinuela, E., Salas, M.L., Salas, J., 1999. Replication of African swine fever virus DNA in infected cells. Virology 257, 524-536.

Salas, J., Salas, M.L., Vinuela, E., 1999. African swine fever virus: a missing link between poxviruses and iridoviruses? In: Domingo, E., Webster, R.G., Holland, J.J. (Eds.), Origin and Evolution of Viruses. Academic Press, London, pp. $467-480$.

Schloer, G.M., 1985. Polypeptides and structure of African swine fever virus. Virus Res. 3, 295-310.

Simon-Mateo, C., Andres, G., Vinuela, E., 1993. Polyprotein processing in African swine fever virus: a novel gene expression strategy for a DNA virus. EMBO J. 12, 2977-2987.

Vinuela, E., 1987. Molecular biology of African swine fever virus. In: Becker, Y. (Ed.), African swine fever. Nijhoff, Boston, MA, pp. 31-49.

Whittaker, G.R., Helenius, A., 1998. Nuclear import and export of viruses and virus genomes. Virology 246, 1-23.

Whittaker, G.R., Kann, M., Helenius, A., 2000. Viral entry into the nucleus. Annu. Rev. Cell Dev. Biol. 16, 627-651.

Whittaker, G.R., 2003. Virus nuclear import. Adv. Drug Deliv. Rev. 55, 733-747.

Yanez, R.J., Vinuela, E., 1993. African swine fever virus encodes a DNA ligase. Virology 193, 531-536.

Yanez, R.J., Rodriguez, J.M., Boursnell, M., Rodriguez, J.F., Vinuela, E., 1993. Two putative African swine fever virus helicases similar to yeast 'DEAH' pre-mRNA processing proteins and vaccinia virus ATPases D11L and D6R. Gene 134, 161-174.

Yanez, R.J., Rodriguez, J.M., Nogal, M.L., Yuste, L., Enriquez, C., Rodriguez, J.F., Vinuela, E., 1995. Analysis of the complete nucleotide sequence of African swine fever virus. Virology 208, 249-278. 